\title{
Gangliosides and CD82 inhibit the motility of colon cancer by downregulating the phosphorylation of EGFR at different tyrosine sites and signaling pathways
}

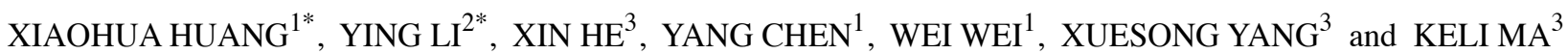 \\ ${ }^{1}$ Department of Clinical Biochemistry, College of Laboratory Medicine, Dalian Medical University, Dalian, \\ Liaoning 116044; ${ }^{2}$ Department of Clinical Laboratory, The Second Affiliated Hospital, Dalian, Liaoning 116023; \\ ${ }^{3}$ Department of Biochemistry and Molecular Biology, Dalian Medical University, Dalian, Liaoning 116044, P.R. China
}

Received December 20, 2019; Accepted April 15, 2020

DOI: $10.3892 / \mathrm{mmr} .2020 .11467$

\begin{abstract}
Previous studies have shown that (GM3), a ganglioside, suppresses hepatoma cell motility and migration by inhibiting phosphorylation of EGFR and the activity of the PI3K/AKT signaling pathway. Therefore, the aim of the present study was to investigate whether the combined treatment of CD82 with gangliosides can exert a synergistic inhibitory effect on cell motility and migration. Epidermal growth factor receptor (EGFR) signaling was studied for its role in the mechanism through which CD82 and gangliosides synergistically inhibit the motility and migration of SW620 human colon adenocarcinoma cells. GM3 and/or GM2 treatment, and/or overexpression of CD82 was performed in SW620 cells. High-performance thin layer chromatography, reverse transcription-quantitative PCR, western blotting and flow cytometry assays were used to confirm the content changes of GM2, GM3 and CD82. In addition, the phosphorylation of EGFR, MAPK and Akt were evaluated by western blot analysis. SW620 cell motility was investigated using wound healing analysis and chemotaxis migration assay. The combination of GM3 and GM2 with CD82 was found to markedly suppress EGF-stimulated SW620 cell motility compared with the individual factors or combination of GM2 or GM3 with CD82 by inhibiting the phosphorylation of EGFR. The results suggested that CD82 in combination with either GM2 or GM3 can exert a synergistic inhibitory effect on cell motility and
\end{abstract}

Correspondence to: Professor Keli Ma, Department of Biochemistry and Molecular Biology, Dalian Medical University, 9 West Section, Lvshun South Road, Dalian, Liaoning 116044, P.R. China

E-mail: makelipaper@163.com

*Contributed equally

Key words: ganglioside, CD82, metastasis, epidermal growth factor receptor, Akt serine/threonine kinase, mitogen-activated protein kinase migration; however, the synergistic mechanisms elicited by GM2 or GM3 with CD82 differ.

\section{Introduction}

Colon cancer is the most frequent malignant tumor of the digestive tract and has a high mortality due to its high metastatic potential. It was responsible for $\sim 1.4$ million new cases and 700,000 deaths worldwide in 2012 (1). A previous study has reported that the loss of CD82 expression and the increased expression of epidermal growth factor receptor (EGFR) and/or its ligands are notable biological characteristics of colon cancer, and these have been associated with an increased risk of metastasis and hence poor prognosis (2). CD82 is a member of the tetraspanin superfamily of transmembrane proteins that are ubiquitously expressed in normal tissues but expressed at low levels in cancer cells and tissues. CD82 exerts its biological effects by regulating cell migration, fusion, adhesion and proliferation (3-5). An inverse relationship exists between CD82 expression and the invasive and metastatic potentials of cancer cells, and this relationship is frequently observed in a wide range of malignant solid tumors, such as gastric, colon, breast, skin, lung, pancreas and liver tumors (6-13). Thus, CD82 is considered a wide-spectrum invasion- and metastasis-suppressor.

CD82 suppresses cancer metastasis primarily by inhibiting cancer cell migration and invasion. In the plasma membrane, CD82 associates with other tetraspanin proteins to form the tetraspanin web $(14,15)$ consequently recruiting some membrane components such as EGFR $(2,16)$, integrins $(17,18)$ and gangliosides (19) into the tetraspanin web. The formation of the tetraspanin web is a prerequisite for the proper functioning of CD82. However, the mechanism through which CD82 redistributes the components of the tetraspanin web and the role of CD82-associated proteins in the CD82-mediated suppression of cell migration remains unclear.

Gangliosides are sialylated glycosphingolipids found in the mammalian cell membrane that are involved in numerous biological functions $(20,21)$. It has been speculated that gangliosides serve a role in a number of cell-surface events, such as cell-to-cell adhesion, cell-extracellular matrix adhesion, and 
plasma membrane receptor-mediated modulation of transmembrane signaling (22-26). There is a growing body of evidence that suggests that CD82 and gangliosides, particularly GM3 (NeuNAc 2 2, 3 Gal $\beta 1,4 \mathrm{Glc1}$, 1Cer) and GM2 (GalNac $\beta 1$, 4[NeuNAc $\alpha 2$, 3] Gal $\beta 1,4 \mathrm{Glc1,}$ 1Cer), which are characteristic components of mammalian cell membranes, can synergistically inhibit cancer cell migration (27-29). Previous studies have related the low expression levels of GM3 to cancer development and invasiveness, while GM2 are abundantly expressed on the cell surface of certain types of human cancer. Although it is known that quantitative and qualitative changes in the expression of gangliosides are involved in oncogenic transformation of cells (30-32), the exact mechanism has not been fully elucidated. The present study aimed to investigate whether EGFR signaling is involved in the mechanism through which CD82 and gangliosides synergistically inhibit the motility and migration of SW620 cells.

\section{Materials and methods}

Cell lines and cell culture. The human colorectal adenocarcinoma cell line SW620 was obtained from the Shanghai Institute of Biochemistry and Cell Biology, Chinese Academy of Sciences. For all experiments, SW620 cells were grown in 10 -cm cell culture dishes or in multiwell plates in L15 medium supplemented with $10 \%$ heat-inactivated FBS (both Thermo Fisher Scientific, Inc.), $100 \mathrm{U} / \mathrm{ml}$ penicillin and $100 \mathrm{mg} / \mathrm{ml}$ streptomycin under a $5 \% \mathrm{CO}_{2}$ atmosphere at $37^{\circ} \mathrm{C}$.

Antibodies and reagents. Immunoglobulin $\mathrm{G}(\mathrm{IgG})$ antibodies against EGFR (cat. no. sc-365829; 1:2,000), phosphorylated (p)-EGFR (Tyr-1173; cat. no. sc-57542; 1:1,000), p-EGFR (Tyr-845) (cat. no. sc-57542; 1:1,000), p-EGFR (Tyr-1045; cat. no. sc-57541; 1:1,000), Akt1/2/3 (cat. no. sc-55523; 1:2,000), p-Akt1/2/3 (Ser473; cat. no. sc-81433; 1:2,000), p-Akt1/2/3 (Thr308; cat. no. sc-16646-R; 1:2,000), MAPK (cat. no. sc-7383; 1:2,000) and p-p44/42 MAPK (cat. no. sc-81492; 1:1,000) were obtained from Santa Cruz Biotechnology, Inc. Anti-CD82 IgG (cat. no. ab59509; 1:5,000) and p-EGFR (Tyr-992; cat. no. ab5638; 1:5,000) were obtained from Abcam, anti-GM3 IgM (cat. no. 303-06541; 1:200) was obtained from Wako Pure Chemical Industries Ltd., and anti-V5 IgG (cat. no. MA5-15253; 1:1,000) was purchased from Invitrogen (Thermo Fisher Scientific, Inc.). Among secondary antibodies, Goat anti-rabbit IgG-horseradish peroxidase (cat. no. sc-2004; $1: 2,000)$, rabbit anti-goat IgG-horseradish peroxidase (cat. no. sc-2768; 1:2,000), goat anti-mouse IgG-horseradish peroxidase (cat.no. sc-2005; 1:2,000) and Alexa Fluor 488-conjugated anti-mouse IgG (cat. no. sc-362257; 1:200) were obtained from Santa Cruz Biotechnology, Inc. FITC-conjugated antimouse IgM was obtained from BD Biosciences Pharmingen (cat. no. 555988; 1:500). Epidermal growth factor (EGF) was obtained from ProSpec-Tany TechnoGene, Ltd., D-threo1-phenyl-2-palmitoyl amino-3-pyrrolidino-1-propanol (P4) was purchased from Matreya, Inc., and GM3 and GM2 were purchased from Sigma-Aldrich (Merck KGaA).

Glycosphingolipid extraction and high-performance thin layer chromatography (HPTLC). In order to decrease the ganglioside expression in SW620 cells, the cells $\left(5 \times 10^{6} /\right.$ dish) were grown in $10-\mathrm{cm}$ dishes at $37^{\circ} \mathrm{C}$ and treated with $1.0 \mu \mathrm{M} \mathrm{P} 4$, a specific inhibitor for UDP-glucose ceramide glucosyltransferase and a key enzyme in the synthesis of glucosylceramide. After $48 \mathrm{~h}$, the cells were harvested by trypsinization and washed three times with PBS. The gangliosides were extracted and analyzed as previously described by Ladisch et al (33). Briefly, the cell pellet was extracted twice with chloroform:methanol $(1: 1, \mathrm{v} / \mathrm{v})$, and the extracts containing the total lipids $(\sim 15 \mathrm{ml})$ were combined and dried under a stream of $\mathrm{N}_{2}$. The gangliosides were purified by partitioning the dried samples in diisopropyl ether: 1-butanol: $17 \mathrm{mM}$ aqueous $\mathrm{NaCl}(6: 4: 5, \mathrm{v} / \mathrm{v})$ followed by Sephadex G-50 gel (Cytiva) filtration and lyophilization. Samples $(20 \mu \mathrm{l})$ were loaded and the individual gangliosides were separated on silica gel 60 HPTLC plates (Merck KGaA) with a solvent system consisting of chloroform:methanol:0.25\% aqueous $\mathrm{CaCl}_{2} \cdot 2 \mathrm{H}_{2} \mathrm{O}(60: 40: 9, \mathrm{v} / \mathrm{v} / \mathrm{v})$. The gangliosides were visualized as purple bands by spraying with resorcinol- $\mathrm{HCl}$ reagent and heating at $120^{\circ} \mathrm{C}$ for $2 \mathrm{~min}$, comparing with the standards including GM3, GM2, GM1 GD1a, GD1b and GT1b.

To increase ganglioside expression in SW620 cells, the cells $\left(5 \times 10^{5} /\right.$ well) were treated with $50 \mu \mathrm{M}$ GM3 for $48 \mathrm{~h}$ before harvesting. Cells were separated with trypsin + EDTA and washed with PBS. Aliquots of cells $\left(1 \times 10^{5}\right)$ were incubated with mouse anti-GM3 IgM (1:200; cat. no. 303-06541, Wako Pure Chemical Industries Ltd.) for $1 \mathrm{~h}$ on ice. Subsequently, cells were washed with PBS, incubated with FITC-conjugated anti-mouse IgM (1:500; cat. no. 555988, BD Biosciences Pharmingen) for $40 \mathrm{~min}$ on ice. The cells were fixed with $2 \%$ paraformaldehyde in PBS and analyzed using a Coulter EPICS XL flow cytometer (Beckman Coulter, Inc.).

Flow cytometry analysis. To identify the location of recombinant CD82, the flow cytometry assay was performed in non-permeable and permeable conditions. Cells were detached with trypsin/EDTA, and $5 \times 10^{4}$ cells were permeabilized by $0.1 \% \mathrm{TX}-100$ in TBS for $15 \mathrm{~min}$, then washed with $1 \%$ BSA/0.1\% NaN 3/PBS. Aliquots of the cells $\left(1 \times 10^{5}\right)$ were incubated with primary antibodies including anti-CD82 (1:1,000; cat. no. ab59509, Abcam) and anti-GM3 (1:200; cat. no. 303-06541, Wako Pure Chemical Industries Ltd.) in $5 \%$ goat serum (Thermo Fisher Scientific, Inc.), 1\% BSA (Thermo Fisher Scientific, Inc.) and $0.1 \%$ NaN3 in PBS for $1 \mathrm{~h}$ on ice, and then with appropriate Alexa Fluor 488-conjugated secondary antibodies for $30 \mathrm{~min}$ on ice. Cells were analyzed using a Coulter EPICS XL flow cytometer and FlowJo software (version 7.6.1; FlowJo LLC).

Transfections. The cDNA of CD82 was amplified from SW480 cells by PCR. CD82 gene was inserted into the pcDNA ${ }^{\mathrm{TM}} 4 /$ V5 plasmid (Thermo Fisher Scientific, Inc.) at the restriction endonuclease sites NheI and XhoI by the use of T4 DNA ligase (Takara Biotechnology Co., Ltd.). The recombinant plasmid was termed pcDNA ${ }^{\mathrm{TM}}$ 4/V5-CD82 and identified by sequencing (Takara Biotechnology Co., Ltd.). The pcDNA ${ }^{\mathrm{TM}} 4 / \mathrm{V} 5-C D 82$ plasmid was constructed and transfected into SW620 cells using Lipofectamine ${ }^{\circledR} 2000$ (Invitrogen; Thermo Fisher Scientific, Inc.) in Opti-MEM medium as OE (overexpression of $C D 82)$, and the empty vector was also transfected as control. The cells $\left(1 \times 10^{5} /\right.$ well) were seeded in 12 -well plate for $24 \mathrm{~h}$ with $1.6 \mu \mathrm{g}$ of the recombinant plasmid and $2 \mu \mathrm{l}$ 
Lipofectamine 2000 diluted with $100 \mu \mathrm{l}$ Opti-MEM medium separately for $5 \mathrm{~min}$ at room temperature. The mixture of diluted plasmid and Lipofectamine 2000 were incubated for $15 \mathrm{~min}$ at room temperature and then added into the well. The transfect cells were analyzed after incubation with the mixture at $37^{\circ} \mathrm{C}$ for $48 \mathrm{~h}$ following the manufacturer's protocol.

Reverse transcription-quantitative PCR (RT-qPCR). The expression of $C D 82$ was measured using RT-qPCR after transfected into SW620 cells for $48 \mathrm{~h}$. Total RNA was extracted and purified using a MiniBEST Universal RNA Extraction kit (Takara Biotechnology Co., Ltd.). The first strand of cDNA was prepared from $2 \mu \mathrm{g}$ RNA using TransScript First-Strand cDNA Synthesis SuperMix (TransGen Biotech Co., Ltd.). qPCR was performed using TransStart Top Green qPCR SuperMix (TransGen Biotech Co., Ltd.) under StepOnePlus system (Applied Biosystems; Thermo Fisher Scientific, Inc.), according to the manufacturer's protocol. The following thermocycling conditions were used for the qPCR: $94^{\circ} \mathrm{C}$ for $30 \mathrm{sec}$; followed by 40 cycles of $94^{\circ} \mathrm{C}$ for $5 \mathrm{sec}, 60^{\circ} \mathrm{C}$ for $15 \mathrm{sec}$ and $72^{\circ} \mathrm{C}$ for $10 \mathrm{sec}$. The following primer pairs were used for the qPCR: $C D 82$ forward, 5'-GCCGACAAGAGCAGTTTCAT-3' and reverse, 5'-GGAAGCCCATGAGCATAGTG-3'; and GAPDH forward, 5'-AGTCCTACCACGATACCAAAGT-3' and reverse, 5'-CATGAGAAGTACGACAACAGCCT-CAT. Relative mRNA expression levels were determined by using the $2^{-\Delta \Delta C q}$ method. To ensure test reliability, cDNA amounts were optimized, the $\mathrm{Cq}$ values were kept below 35 , and mRNA expression levels normalized to $G A P D H$ as the reference gene.

Western blotting. For western blot analysis, cells were harvested and lysed in RIPA buffer ( $1 \%$ Triton X-100, $150 \mathrm{mM}$ $\mathrm{NaCl}, 25 \mathrm{mM}$ Tris, $\mathrm{pH} 7.5,0.5 \%$ sodium deoxycholate, $0.1 \%$ SDS, $5 \mathrm{mM}$ pyrophosphate, $50 \mathrm{mM} \mathrm{NaF}$ ) containing $1 \mathrm{mM}$ $\mathrm{Na}_{3} \mathrm{VO}_{4}, 1 \mathrm{mM}$ DTT, $1 \%$ protease inhibitor cocktail and $1 \%$ phosphatase inhibitor cocktail. Protein concentration was determined by using a bicinchoninic acid assay and $10 \mu \mathrm{g}$ protein were separated by SDS-PAGE on $12 \%$ polyacrylamide gels. The resolved proteins were transferred to PVDF membranes (EMD Millipore) and incubated with primary antibodies against EGFR (1:2,000; cat. no. sc-365829, Santa Cruz Biotechnology, Inc.), p-EGFR (Tyr-1173; 1:1,000; cat. no. sc-57542, Santa Cruz Biotechnology, Inc.), p-EGFR (Tyr845; 1:1,000; cat. no. sc-57542, Santa Cruz Biotechnology, Inc.), p-EGFR (Tyr-1045; 1:1,000; cat.no. sc-57541, Santa Cruz Biotechnology, Inc.), Akt1/2/3 (1:2,000; cat. no. sc-55523, Santa Cruz Biotechnology, Inc.), p-Akt1/2/3 (Ser473; 1:2,000; cat. no. sc-81433, Santa Cruz Biotechnology, Inc.), p-Akt1/2/3 (Thr308; 1:2,000; cat. no. sc-16646-R, Santa Cruz Biotechnology, Inc.), MAPK (1:2,000; cat. no. sc-7383, Santa Cruz Biotechnology, Inc.), p-p44/42MAPK (1:1,000; cat. no. sc-81492, Santa Cruz Biotechnology, Inc.), p-EGFR (Tyr-992; 1:5,000; cat. no. ab5638, Abcam) and CD82 IgG (1:5,000; cat. no. ab59509, Abcam). After washing with TBST $(0.05 \%$ Tween-20), the membranes were incubated with goat anti-rabbit IgG-horseradish peroxidase $(1: 2,000$; cat. no. sc-2004, Santa Cruz Biotechnology, Inc.) and rabbit anti-goat IgG-horseradish peroxidase $(1: 2,000$; cat. no. sc-2768, Santa Cruz Biotechnology, Inc.). Protein bands were visualized using the ECL kit (Cytiva) and images captured using the VersaDoc ${ }^{\mathrm{TM}}$ Imaging system (Bio-Rad Laboratories, Inc.). Densitometric semi-quantification of protein expression levels was performed using the Gel-Pro analyzer v4.0 (Media Cybernetics, Inc.) and GraphPad Prism 5 (GraphPad Software, Inc.) with $\beta$-actin as the loading control.

Wound healing assay. The invasive capacity of the SW620 cells was verified through a wound healing assay. SW620 cells were seeded on 6-well plates at a cell density of $2 \times 10^{6}$ cells/well. SW620 cells were treated with P4 and/or GM3 and/or transfected as OE or empty vector as control, aforementioned. After incubating the cells overnight in serum-free medium, the scratch was made when the cells reached $85 \%$ confluence. Cells were washed three times with PBS and incubated in serum-free medium containing $10 \mathrm{ng} / \mathrm{ml}$ EGF for $48 \mathrm{~h}$ at $37^{\circ} \mathrm{C}$. Images of the migrated cells were captured using a light microscope at x200 magnification (Olympus Corporation) in three randomly selected fields, wound closure was measured with ImageJ software (version $1.47 \mathrm{v}$, National Institutes of Health).

Transwell migration assay. For the Transwell migration assay, cells were treated as aforementioned and subsequently incubated in serum-free medium overnight at $37^{\circ} \mathrm{C}$. Cells $\left(1 \times 10^{5}\right)$ were then resuspended in $300 \mu \mathrm{l}$ of serum-free medium containing $10 \mathrm{ng} / \mathrm{ml}$ EGF and placed on the top compartment of a chamber with $8-\mu \mathrm{m}$ pore polycarbonate filters. The bottom chamber was filled with $250 \mu \mathrm{l}$ medium with $10 \%$ FBS. After $48 \mathrm{~h}$, cells that migrated to the lower surface of the membrane were fixed with methanol for $20 \mathrm{~min}$ at room temperature and stained with $0.1 \%$ DAPI; images were captured from five randomly chosen fields, and cells were counted under a light microscope at x100 magnification (Olympus Corporation). To block MAPK and PI3K/Akt pathways with U0126 and LY294002 inhibitors, the SW620 cells were exposed to $10 \mu \mathrm{M}$ of U0126 or $15 \mathrm{mM}$ of LY294002 for $4 \mathrm{~h}$ at $37^{\circ} \mathrm{C}$. The cells were then harvested and used for migration assay.

Statistical analysis. All experiments were repeated at least three times and they consistently yielded similar results. The data were analyzed using GraphPad Prism 5 (GraphPad Software, Inc.). Unpaired t-test or one-way ANOVA followed by Dunnett's post hoc test were used for statistical comparisons between groups. The results are expressed as the mean \pm standard deviation. $\mathrm{P}<0.05$ was considered to indicate a statistically significant difference.

\section{Results}

Alteration of ganglioside content and overexpression of CD82 in SW620 Cells. To investigate the role of gangliosides in SW620 cells, which may be related to a metastatic ability (34), the expression of gangliosides in SW620 cells was altered by inhibiting their synthesis through treatment with $\mathrm{P} 4$ and by the addition of exogenous GM3. The cells were treated with $1 \mu \mathrm{M} \mathrm{P} 4$ for $48 \mathrm{~h}$, and the gangliosides were then extracted and monitored using HPTLC. The results showed that SW620 cells predominantly contained GM2 and that the gangliosides found in SW620 cells were nearly depleted with $1 \mu \mathrm{M}$ P4 (Fig. 1A). 

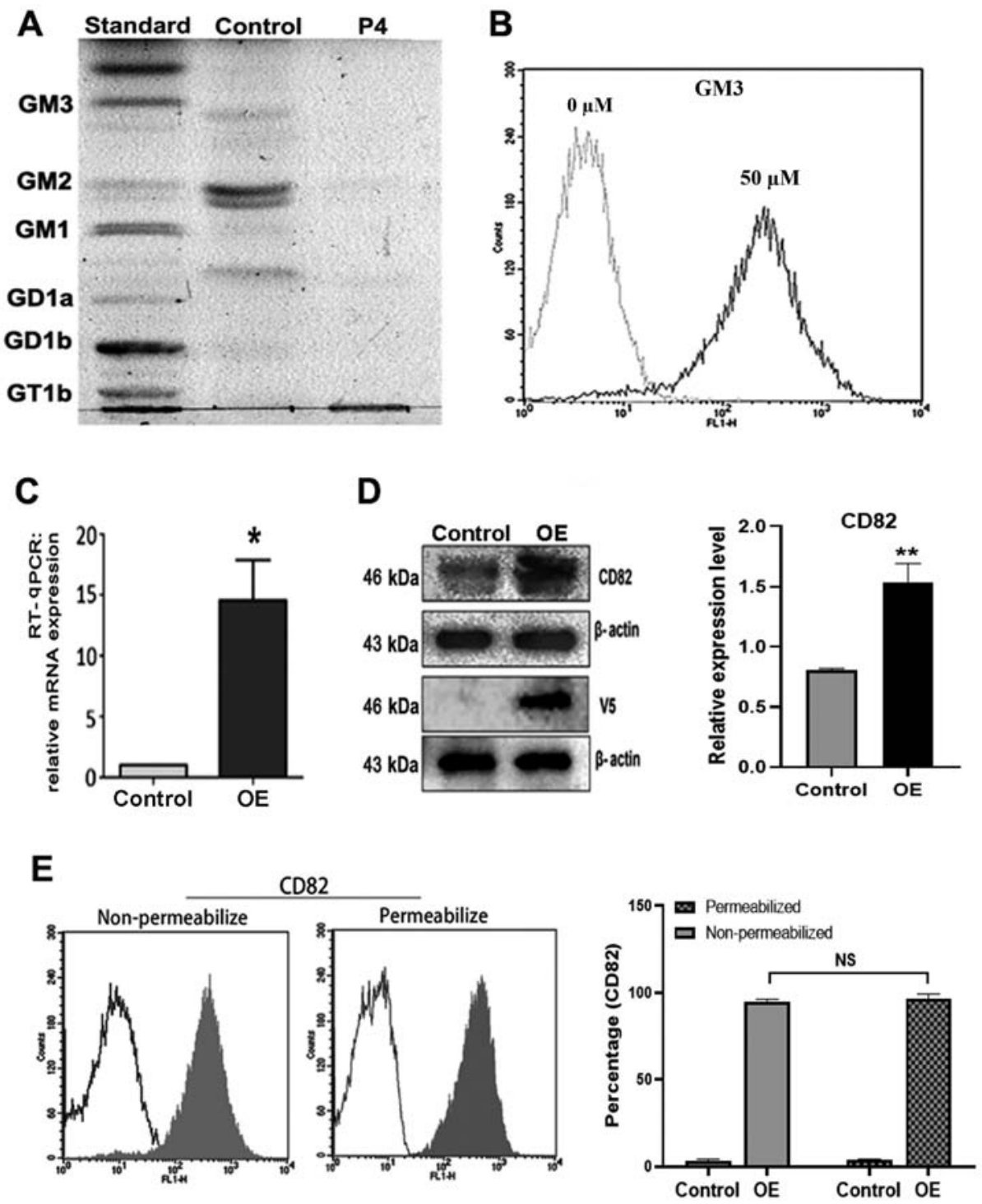

Figure 1. Expression of gangliosides and CD82 in cells. (A and B) Cells were treated with either $1.0 \mu \mathrm{M} \mathrm{P} 4$ or $50 \mu \mathrm{M} \mathrm{GM}$. After $48 \mathrm{~h}$ of incubation, the gangliosides were extracted and assayed by (A) HPTLC and (B) flow cytometry. (C and D) pcDNA4/V5-CD82 or pcDNA4.0 (control) plasmids were constructed and transfected into SW620 cells, and CD82 mRNA and protein expression levels were measured by (C) RT-qPCR and (D) western blot, respectively. Expression of V5- tagged CD82 protein was also detected by western blot; $\beta$-actin was used as a loading control. (E) Flow cytometric analysis of CD82 expression after cell transfection. Shaded areas, SW620 cells transfected with pcDNA4/V5-CD82; non-shaded areas, SW620 cells transfected with pcDNA4.0 control. All the results shown are representative of three independent experiments. Data are presented as the mean \pm standard deviation from three independent experiments. ${ }^{*} \mathrm{P}<0.05$ and ${ }^{* *} \mathrm{P}<0.01$ vs. Control group. CD82, CD82 antigen; GD1a and GD1b, disialoganglioside; GM, monosialodihexosylganglioside; Ganglioside GT1b; P4, D-threo-1-phenyl-2-palmitoyl amino-3-pyrrolidino-1-propanol; RT-qPCR, reverse transcription-quantitative PCR; OE, overexpression of wild-type CD82.

To increase the GM3 content, cells were treated with $50 \mu \mathrm{M}$ exogenous GM3 for $48 \mathrm{~h}$, and the expression of GM3 in the cells was determined using flow cytometry (Fig. 1B).

To investigate the influence of CD82 on SW620 cell motility and migration, the cells were transfected with pcDNA4/V5-CD82 as OE or pcDNA4.0 empty vector as control, and the mRNA and protein expression levels of CD82 was measured using RT-qPCR and western blotting, respectively (Fig. 1C and D). The pcDNA4/V5-CD82 plasmid also contained the fusion proteins including CD82 and V5 epitopes. To confirm the overexpression of CD82, the fusion protein was detected by western blot with V5 antibody (Fig. 1D), and the CD82 expression profiles were also compared using flow cytometry (Fig. 1E). The results demonstrated that CD82 and V5 expression were significantly higher in OE of SW620 cells compared with the control. To identify the location of recombinant CD82, the flow cytometry assay was performed in non-permeable and permeable conditions. CD82 expression was observed in 94.9 and $96.6 \%$ of CD82 transfected cells, but only in 3.4 and $3.6 \%$ of control cells, without or with permeabilization, respectively (Fig. 1E). No notable difference in the expression of CD82 was observed between non-permeabilized and permeabilized cells transfected with pcDNA4/V5-CD82 plasmid, which indicated that the overexpression of CD82 was mainly on the cell surface.

Effect of gangliosides and CD82 on the motility and migration of EGF-stimulated SW620 cells in vitro. To understand the influence of gangliosides and CD82 on the metastatic ability of SW620 cells, in vitro cell motility and migration 

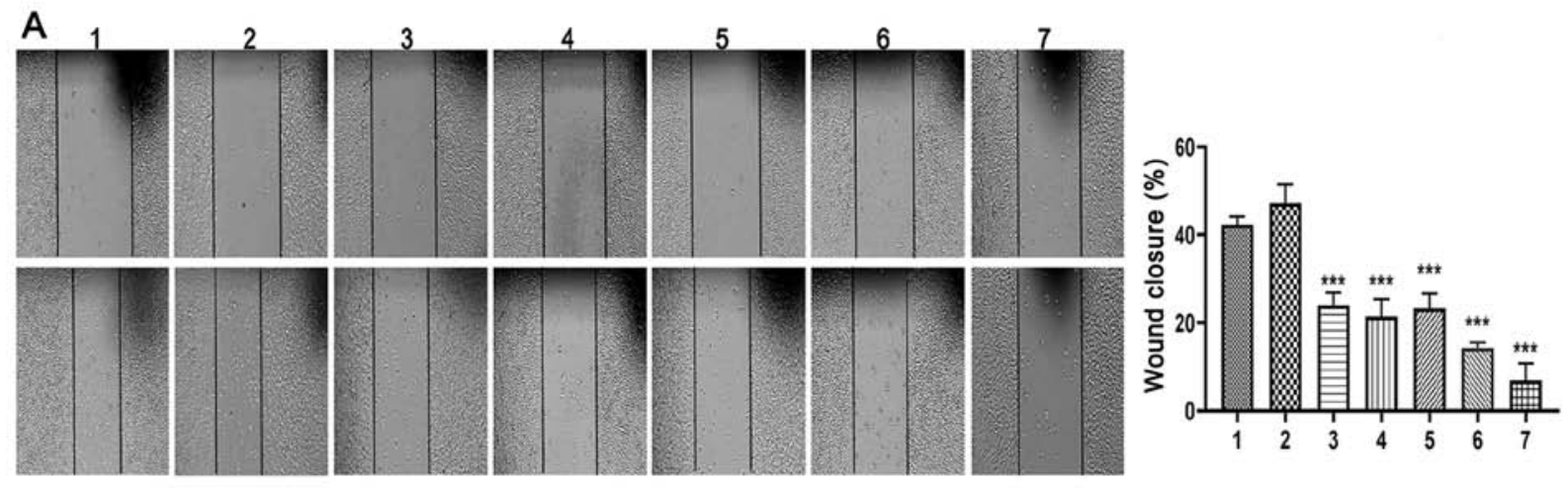

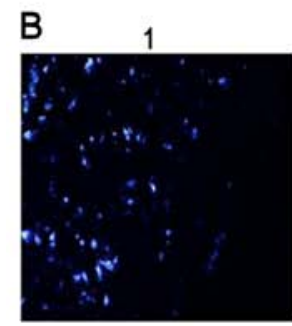

5

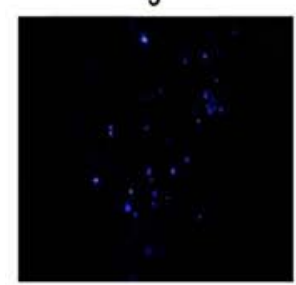

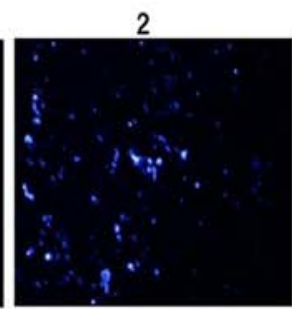

6

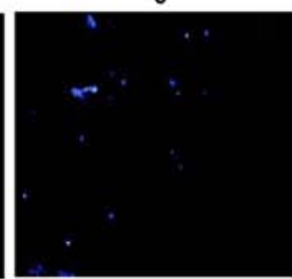

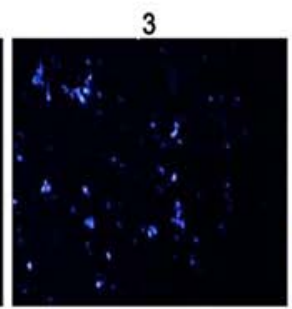

7

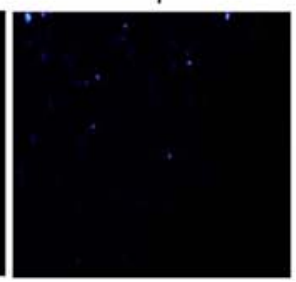

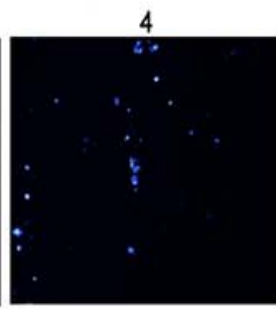

1.P4+control

2.Control

3.P4+GM3+Control

4.P4+OE

$5 . \mathrm{OE}$

6.P4+GM3+OE

7.GM3+OE

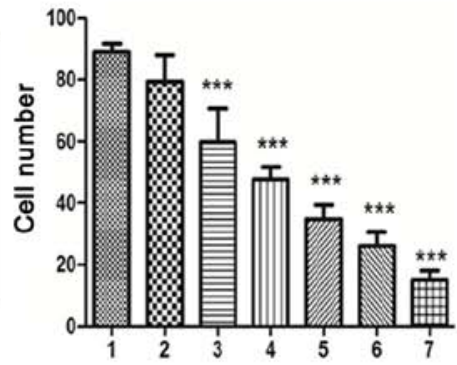

Figure 2. Effect of gangliosides and CD82 on the epidermal growth factor-induced migration of SW620 cells in vitro. (A and B) SW620 cells were cultured and treated and/or transfected with the indicated agents and plasmids for $48 \mathrm{~h}$. Cell migration assays were performed using (A) wound healing analysis and (B) chemotaxis migration assays. For wound healing assay, the widths of wound closure were measured (magnification, x200). For Transwell assay, the average number of cells that migrated through the filter were counted (magnification, x100). Data are presented as the mean \pm SD from three independent experiments. ${ }^{* * * *} \mathrm{P}<0.001$ vs. $\mathrm{P} 4+\mathrm{Control}$ group. The expression of ganglioside and CD82 in each group are shown as following, 1. No expression of GM2/GM3 and CD82, 2. Only expression of GM2, 3. Only expression of GM3, 4. Only expression of CD82, 5. Expression of GM2 and CD82, 6. Expression of GM3 and CD82, 7. Expression of GM2, GM3 and CD82. GM3, monosialodihexosylganglioside GM3; P4, D-threo-1-phenyl-2-palmitoyl amino-3-pyrrolidino-1-propanol; OE, overexpression of wild-type CD82

assays were performed using both wound healing analysis and Transwell migration assay. Each group, stimulated with EGF, was compared with the P4 treatment control cells transfected with an empty vector, which had low expression of gangliosides and CD82 (Fig. 2A1 and B1). GM2 downregulation in SW620 cells had no significant effect on cell motility and migration in vitro compared with the control cells (Fig. 2A1, A2, B1 and B2). However, increasing GM3 inhibited cell motility and migration in vitro (Fig. 2A3 and B3). CD82 alone or in combination with GM2 significantly inhibited cell motility and migration in vitro (Fig. 2A4, B4, A5 and B5). GM3 overexpression further enhanced the inhibitory effect of CD82 (Fig. 2A6 and B6). The combination of GM3 overexpression and uninhibited GM2 expression (that is, without P4 treatment) markedly enhanced the inhibitory effect of CD82 compared with either GM3 or GM2 alone (Fig. 2A7 and B7). These results suggested that single factor of GM3 or CD82 and combination of CD82 with GM2 or GM3 can inhibit the EGF-stimulated SW620 cell motility and that GM3, GM2 and CD82 in triple combination can show the most effect of inhibition of the EGF-stimulated SW620 cell motility in vitro.
Effect of gangliosides and CD82 on the phosphorylation of EGFR and EGFR-mediated intracellular signaling pathways in EGF-stimulated SW620 cells. To investigate the effects of gangliosides and CD82 on the EGF-stimulated cell motility and migration in vitro, the influence of gangliosides and CD82 on the activity of EGFR was investigated by detecting the phosphorylation levels of three of its tyrosine residues, all the experimental groups were compared with the first group, which had low expression of gangliosides and CD82. Whereas GM2 could not inhibit the phosphorylation at the Tyr1173, Tyr1045 and Tyr845 residues (Fig. 3A; lane 2), GM3 could significantly inhibit the EGF-stimulated phosphorylation levels at the Tyr1173 and Tyr1045 residues (Fig. 3A; lane 3), and CD82 could significantly inhibit the phosphorylation levels at the Tyr1045 residue (Fig. 3A; lane 4). The combination of GM3 and CD82 significantly inhibited the phosphorylation of EGFR at both the Tyr1173 and Tyr1045 residues (Fig. 3A; lane 6), and the combination of GM2 and CD82 notably inhibited the phosphorylation of EGFR at the Tyr1045 residue (Fig. 3A; lane 5). The combination of GM3, GM2 and CD82 resulted in the most effective inhibition of phosphorylation levels at Tyr1173, Tyr1045 and Tyr845 residues compared with 
A
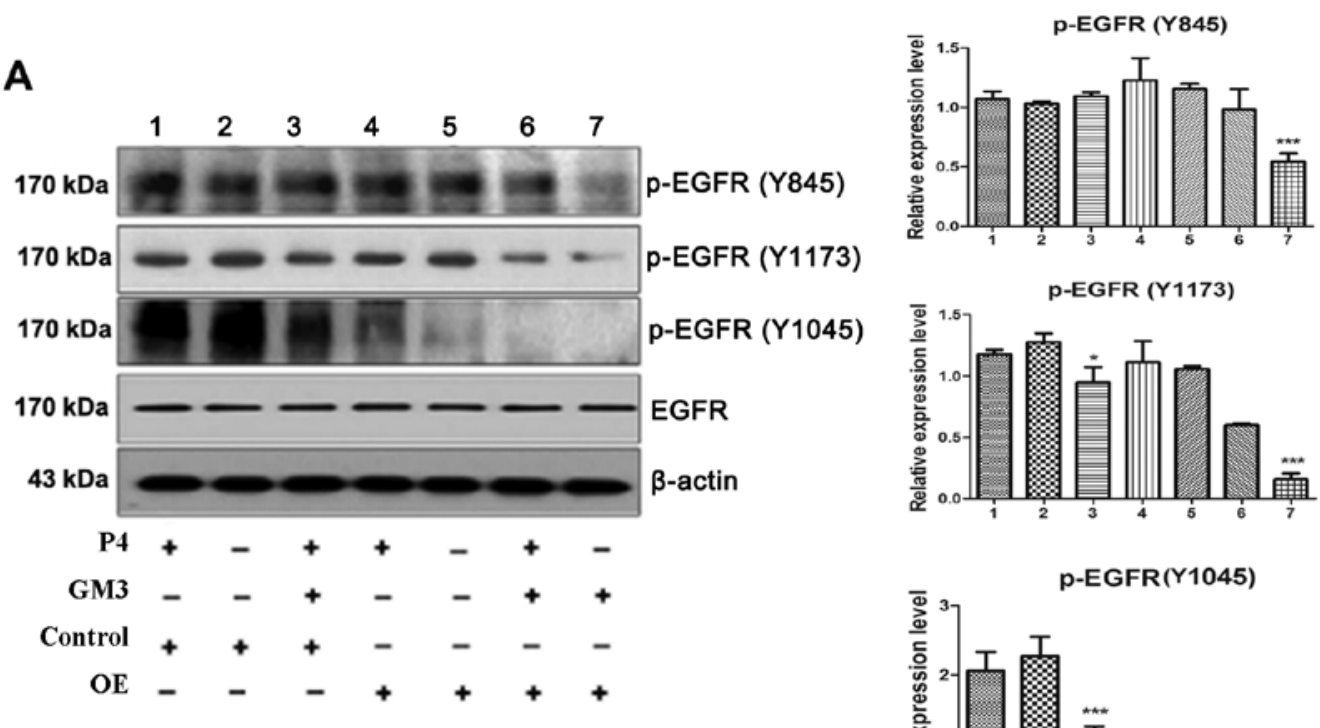

B
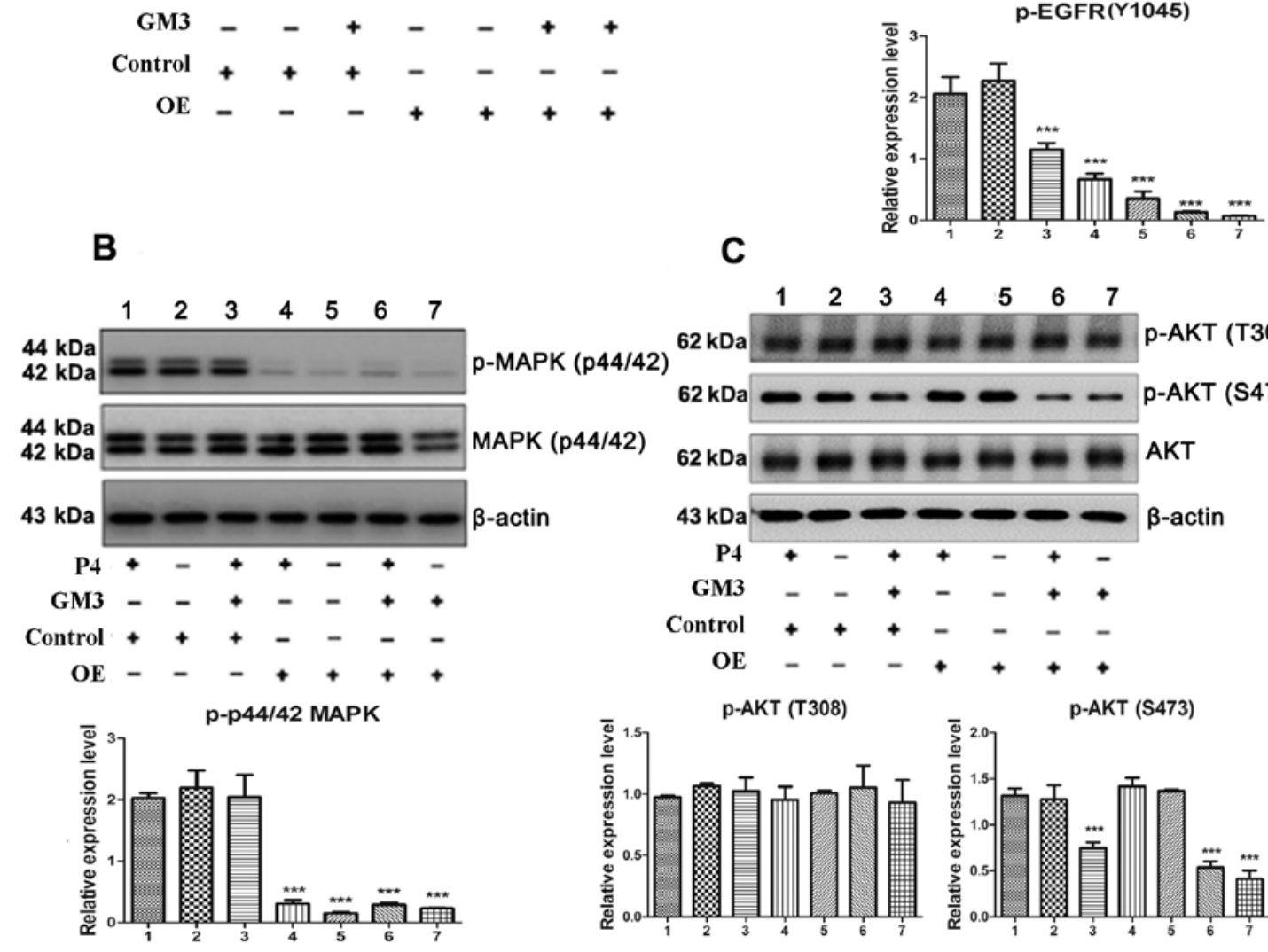

C
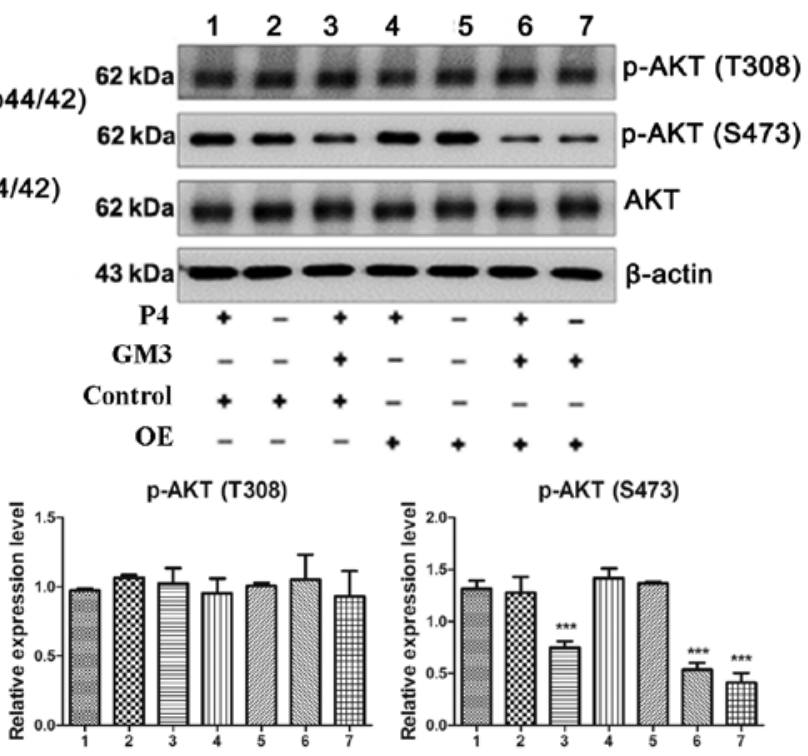

Figure 3. Effects of gangliosides and CD82 antigen on the EGF-induced phosphorylation of EGFR and phosphorylation of Akt and MAPK. (A-C) SW620 cells were cultured and/or transfected with the indicated agents and plasmids for $48 \mathrm{~h}$. After starvation, the cells were stimulated with $50 \mathrm{ng} / \mathrm{ml} \mathrm{EGF}$ for $10 \mathrm{~min}$ at room temperature. Protein lysates were prepared and subjected to western blot analysis for the indicated proteins. (A) The phosphorylation of EGFR, (B) phosphorylation of Akt and (C) MAPK. Data are presented as the mean \pm SD from three independent experiments. ${ }^{*} \mathrm{P}<0.05$ and ${ }^{* * * *} \mathrm{P}<0.001$ vs. $\mathrm{P} 4+\mathrm{Control}$ group. EGF, epidermal growth factor; EGFR, epidermal growth factor receptor; GM3, monosialodihexosylganglioside GM3; MAPK, mitogen-activated protein kinase; p-, phosphorylated; P4, D-threo-1-phenyl-2-palmitoyl amino-3-pyrrolidino-1-propanol; OE, overexpression of wild-type CD82.

the control group (Fig. 3A; lane 7). In addition, EGFR phosphorylation levels at the Tyr992 residue was also measured and no obvious difference among the groups was detected (data not shown). These results indicated that gangliosides and CD82 downregulated the activity of EGFR by inhibiting the phosphorylation of different tyrosine residues of EGFR and that the effects of different gangliosides in combination with CD82 are also different.

The EGFR-mediated intracellular signaling pathways that are involved in the regulation of metastasis were also studied. A previous study indicated that MAPK and PI3K/Akt signaling pathways are associated with extracellular and intracellular cues to control cell motility (34). To detect the activity of the MAPK signaling pathway, the phosphorylation levels of MAPK were determined. The results show that neither GM2 nor GM3 influenced the EGF-stimulated phosphorylation of MAPK (Fig. 3B; lanes 2 and 3, respectively), whereas CD82 significantly inhibited the EGF-stimulated phosphorylation of MAPK (Fig. 3B; lane 4). CD82 with GM2 or GM3 (Fig. 3B; lanes 5, and 6) or the mixture of GM2 and GM3 (Fig. 3B; lane 7) also showed notable inhibitory effect on the phosphorylation of MAPK.

To detect the activity of the PI3K/Akt signaling pathways, the phosphorylation levels of Akt were determined. The results showed that GM3, but not GM2, can inhibit the EGF-stimulated phosphorylation of Akt at Ser473 (Fig. 3C; 
A

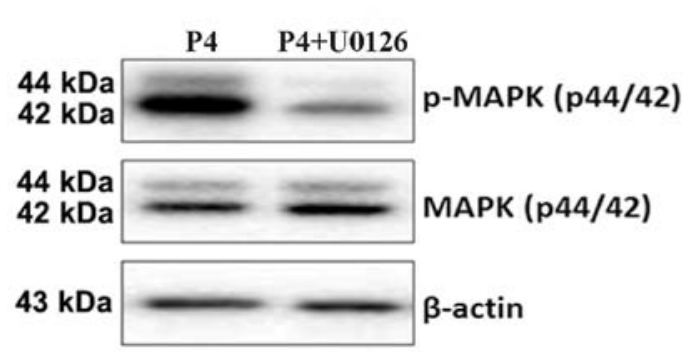

B

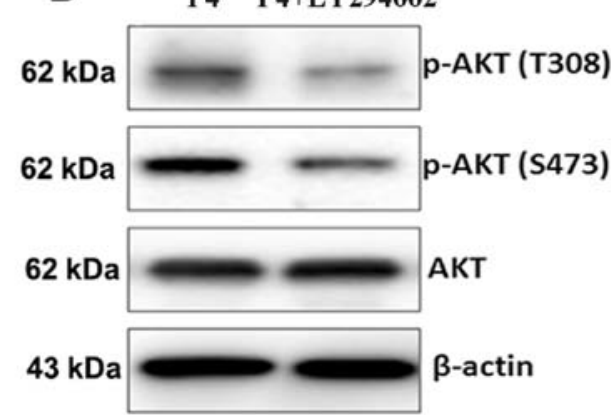

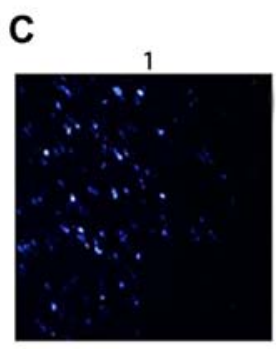
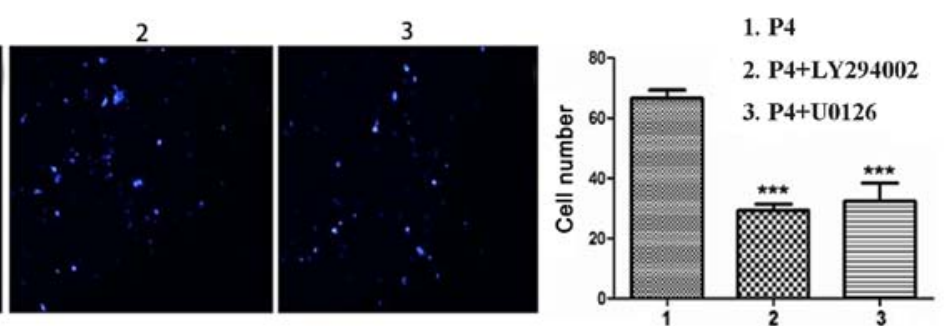

Figure 4. Effect of MAPK and PI3K/Akt signaling pathways inhibition on SW620 cell migration in vitro. To validate that the MAPK and PI3K/Akt signaling pathways are involved in the regulation of SW620 cell motility and migration, MAPK and PI3K/Akt signaling pathways were inhibited with U0126 and LY294002, respectively. The activation of (A) MAPK and (B) PI3K/Akt signaling pathways were assessed by western blotting. (C) After the treatment, cells were harvested, the cell migration was assessed through the chemotaxis migration assay (magnification, $\mathrm{x} 100$ ). The data represent the means $\pm \mathrm{SD}$ from three independent experiments. ${ }^{* * *} \mathrm{P}<0.001$ vs. $\mathrm{P} 4+$ Control group. MAPK, mitogen-activated protein kinase; $\mathrm{p}$-, phosphorylated; P4, D-threo-1-phenyl-2-palmitoyl amino-3-pyrrolidino-1-propanol.

lanes 3 and 2, respectively) and that CD82 alone cannot inhibit the phosphorylation of Akt at Ser473 or T308 (Fig. 3C, lane 4). In addition, CD82 with GM3 but not with GM2 can exert a synergistic inhibitory effect on the EGF-stimulated phosphorylation of Akt at Ser473 (Fig. 3C; lanes 6 and 5, respectively). Addition of exogenous GM3 with overexpression of CD82 notably reduced the EGF-induced phosphorylation of Akt at both Ser473 comparing to the group of exogenous GM3 alone (Fig. 3C; lanes 3 and 6). The mixture of GM3 and GM3 with CD82 also presented the notable inhibitory action on the EGF-stimulated phosphorylation of Akt at Ser473 (Fig. 3C; lane 7).

Effect of blocking the MAPK and PI3K/Akt pathways on cell motility and migration. To validate whether EGF-stimulated cell motility and migration are mainly regulated by the MAPK and PI3K/Akt signaling pathways, the activities of MAPK and PI3K/Akt in the cells were blocked with U0126 and LY294002 inhibitors (Fig. 4). Blocking of the MAPK signaling pathway with U0126 inhibited the phosphorylation of MAPK (Fig. 4A) and inhibited cell motility and migration (Fig. 4C). Blocking of the PI3K/Akt signaling pathway with LY294002 inhibited the phosphorylation of Akt (Fig. 4B) and inhibited the EGF-stimulated cell motility and migration (Fig. 4C). These results show that both the MAPK and PI3K/Akt pathways might be involved in the modulation of SW620 cell motility and migration in vitro.

\section{Discussion}

Colorectal cancer is a highly malignant tumor of the digestive tract, and its incidence and mortality rates are gradually increasing; colorectal cancer has a high mortality rate owing to its high metastatic ability (1). Therefore, the elucidation of the mechanisms involved in metastasis will allow for the selection of anti-metastasis drugs contributing to a lower mortality rate. SW620 cells were originally isolated from the lymph node metastatic foci of a patient with rectal cancer with high lymphatic metastasis ability (35). The loss of CD82 expression and the increased expression of EGFR and/or its ligands are the most notable biological characteristics of colon cancer (7). These features have been associated with an increased risk of metastasis, but their roles in the lymph node metastatic processes have not been completely elucidated.

CD82 is considered a wide-spectrum invasion- and metastasis-suppressor. A number of studies have reported that the combination of CD82 with gangliosides can exert a synergistic inhibitory effect on cell motility and migration $(25,29)$; CD82 and gangliosides can inhibit the phosphorylation and activity of EGFR. The present study aimed to understand the precise mechanism by which CD82 and gangliosides influence EGF-stimulated motility of SW620 cells with potential lymph node metastasis (35) by investigating whether EGFR signaling is involved in the mechanism by which CD82 and gangliosides synergistically inhibit the motility and migration of SW620 cells. The cells were starved overnight and then stimulated with EGF instead of using siEGFR or EGFR selective inhibitors. The results demonstrated that SW620 cells mainly express ganglioside GM2 and low levels of CD82. Moreover, the present study investigated the effects of gangliosides and CD82 on the migration of SW620 cells in vitro by altering the contents of GM2 and GM3 and the expression of CD82 in these cells. The results showed that CD82 can suppress EGF-stimulated SW620 cell motility and migration and that 
GM3, but not GM2, can suppress the EGF-stimulated SW620 cell motility and migration. The results of wound healing and migration assays in vitro indicated that CD82 with either GM3 or GM2 can exert a synergistic inhibitory effect on cell migration, and the mixture of CD82 with both GM2 and GM3 presented the most notable synergistic inhibitory action.

Both GM3 and CD82 can inhibit the phosphorylation and activity of EGFR. In order to understand whether CD82 and gangliosides synergistically inhibit the motility and migration of SW620 cells and whether they are associated with EGFR activity, the present study investigated the effects of CD82 and the gangliosides GM2 and GM3 on the phosphorylation of EGFR and found that gangliosides and CD82 downregulated the activity of EGFR through different mechanisms. GM3 had an inhibitory effect on EGFR activation by inhibiting the phosphorylation of EGFR at the Tyr1045 and Tyr1173 residues, whereas CD82 downregulated the activity of EGFR by inhibiting its phosphorylation at the Tyr1045 residue. The current study also found that CD82 with either GM2 or GM3 can exert a synergistic inhibitory effect on the activity of EGFR, but the mechanisms responsible for the observed synergistic effects are different. GM3 enhanced the inhibitory effect of CD82 on the phosphorylation of EGFR at both the Tyr1045 and Tyr1173 residues, whereas GM2 enhanced the inhibitory effect of CD82 on the phosphorylation of EGFR only at the Tyr1045 residue.

Two intracellular signaling pathways, considered as the important pathways involved in modulation of tumor metastasis $(36,37)$, which can be stimulated by EGFR were analyzed. The overexpression of CD82 inhibited the phosphorylation of MAPK. An increase in the level of GM3 inhibited the phosphorylation of Akt. GM2 cannot influence the phosphorylation of MAPK and Akt but can promote the inhibitory effect of CD82 on the phosphorylation of MAPK. These results indicate that a single factor of GM3 or CD82 inhibits SW620 cell migration only by downregulating either of PI3K or MAPK signaling pathway, whereas the combination of GM3 and CD82 can affect both PI3K and MAPK signaling pathways to decrease cell migration. The most prominent inhibitory effect shows when accumulation of PI3K and MAPK signaling pathways with mixture of GM2, GM3 and CD82. In conclusion, the gangliosides and CD82 inhibited SW620 cell motility and migration through different mechanisms. GM3 suppressed SW620 cell motility and migration by inhibiting the EGF-stimulated phosphorylation of EGFR at the Tyr1045 and Tyr1173 residues and the downregulation of the MAPK signaling pathway, whereas CD82 suppressed SW620 cell motility and migration by inhibiting the EGF-stimulated phosphorylation of EGFR at the Tyr1045 residue and downregulation of the MAPK signaling pathway. The combination of GM3, GM2 and CD82 also resulted in inhibition of phosphorylation levels at Tyr845 residues, which is not an autophosphorylation site. Phosphorylation of EGFR tyrosine 845 is a direct substrate of Src, which is reported as synergism with EGFR through MAPK signaling pathway (38). Thereby, it was hypothesized that Tyr845 may be also important in activation of MAPK pathway and cell migration.

CD82 with either of the gangliosides (GM3 and GM2) exerts a synergistic inhibitory effect; however, the mechanisms are different. GM3 enhanced the inhibitory effect of CD82 on the phosphorylation of EGFR at both the Tyr1045 and Tyr1173 residues and downregulated the PI3K and MAPK signaling pathways, whereas GM2 enhanced the inhibitory effect of CD82 on the phosphorylation of EGFR at only the Tyr1045 residue and downregulated the MAPK signaling pathway. CD82 inhibition of tumor cell movement might depend on gangliosides by promoting internalization of EGFR, which results from phosphorylation of EGFR at specific tyrosine residues to activation of EGFR (39). The internalized EGFR redistributing into distinct cellular compartments causes attenuation of EGFR signaling. Signaling by receptor tyrosine kinases (RTK) is mediated by alternative interactions between specific phosphotyrosine residues in the activated EGFR and individual Src homology 2 (SH2) domains of cytoplasmic effectors. The multiple SH2-containing signal transducers include phosphatidylinositol 3-kinase and Grb2, leading to activation of downstream kinases including PI3K and MAPK signaling pathways. Other studies have revealed that association of a particular receptor with a tetraspanin-enriched microdomains may lead either to the enhancement or attenuation of its activity, and ganglioside expression levels clearly effect the ability of tetraspanins to regulate RTK signaling. In addition, it was also observed that formation of heterotypic GSL-GSL and GSL binding to a GSL-binding protein could affect cellular phenotype by activation of a signal transducer (26). Hakomori (26) reported that non-malignant HCV29 cells with GM2, GM3, and CD82 expression show less motility and invasiveness than invasive YTS1 cells without CD82 expression by regulating c-Met kinase activity. The present study and these previous studies indicated that tetraspanin and specific ganliosides in membrane microdomains may influence signaling activation or block signaling responses by target growth factor receptors. Taken together, the gangliosides and CD82 inhibited SW620 cell motility and migration by different mechanisms. Future investigation of this possibility will help clarify the synergistic role of gangliosides and CD82 on EGFR to inhibit cell motility and invasion.

\section{Acknowledgements}

Not applicable.

\section{Funding}

The present study was supported by The National Natural Science Foundation of China (grant no. 81602546 to X.H. Huang and grant no. 81472716 to K.L. Ma).

\section{Availability of data and materials}

The datasets used and/or analyzed during the current study are available from the corresponding author on reasonable request.

\section{Authors' contributions}

$\mathrm{KM}$ and $\mathrm{XH}$ contributed to the conception and design of the study. XH, YL and KM drafted the manuscript. YC and WW performed the experiments. YL and SY analyzed and interpreted the data. KM and $\mathrm{XH}$ were involved in revising the manuscript. All authors read and approved the final manuscript. 


\section{Ethics approval and consent to participate}

Not applicable.

\section{Patient consent for publication}

Not applicable.

\section{Competing interests}

The authors declare that they have no competing interests.

\section{References}

1. Kolligs FT: Diagnostics and Epidemiology of Colorectal Cancer Visc Med 32: 158-164, 2016

2. Odintsova E, Voortman J, Gilbert E and Berditchevski F: Tetraspanin CD82 regulates compartmentalisation and ligandinduced dimerization of EGFR. J Cell Sci 116: 4557-4566, 2003.

3. Maecker HT, Todd SC and Levy S: The tetraspanin superfamily: Molecular facilitators. FASEB J 11: 428-442, 1997.

4. Pique C, Lagaudrière-Gesbert C, Delamarre L, Rosenberg AR, Conjeaud $\mathrm{H}$ and Dokhélar MC: Interaction of CD82 tetraspanin proteins with HTLV-1 envelope glycoproteins inhibits cell-tocell fusion and virus transmission. Virology 276: 455-465, 2000

5. Sigala S, Faraoni I, Botticini D, Paez-Pereda M, Missale C, Bonmassar E and Spanol P: Suppression of telomerase, reexpression of KAI1, and abrogation of tumorigenicity by nerve growth factor in prostate cancer cell lines. Clin Cancer Res 5: 1211-1218, 1999.

6. Hinoda Y, Adachi Y, Takaoka A, Mitsuuchi H, Satoh Y, Itoh F, Kondoh Y and Imai K: Decreased expression of the metastasis suppressor gene KAI1 in gastric cancer. Cancer Lett 129: 229-234, 1998.

7. Lombardi DP, Geradts J, Foley JF, Chiao C, Lamb PW and Barrett JC: Loss of KAI1 expression in the progression of colorectal cancer. Cancer Res 59: 5724-5731, 1999.

8. Yang X, Welch DR, Phillips KK, Weissman BE and Wei LL: KAI1, a putative marker for metastatic potential in human breast cancer. Cancer Lett 119: 149-155, 1997.

9. Geradts J, Maynard R, Birrer MJ, Hendricks D, Abbondanzo SL, Fong KM, Barrett JC and Lombardi DP: Frequent loss of KAI1 expression in squamous and lymphoid neoplasms. An immunohistochemical study of archival tissues. Am J Pathol 154: 1665-1671, 1999.

10. Adachi M, Taki T, Ieki Y, Huang CL, Higashiyama M and Miyake M: Correlation of KAI1/CD82 gene expression with good prognosis in patients with non-small cell lung cancer. Cancer Res 56: 1751-1755, 1996

11. Friess H, Guo XZ, Tempia-Caliera AA, Fukuda A, Martignoni ME, Zimmermann A, Korc M and Büchler MW: Differential expression of metastasis-associated genes in papilla of vater and pancreatic cancer correlates with disease stage. J Clin Oncol 19: 2422-2432, 2001

12. Sun HC, Tang ZY, Zhou G and Li XM: KAI1 gene expression in hepatocellular carcinoma and its relationship with intrahepatic metastases. J Exp Clin Cancer Res 17: 307-311, 1998.

13. Guo XZ, Friess H, Di Mola FF, Heinicke JM, Abou-Shady M, Graber HU, Baer HU, Zimmermann A, Korc M and Büchler MW: KAI1, a new metastasis suppressor gene, is reduced in metastatic hepatocellular carcinoma. Hepatology 28: 1481-1488, 1998.

14. Hemler ME: Tetraspanin proteins mediate cellular penetration, invasion, and fusion events and define a novel type of membrane microdomain. Annu Rev Cell Dev Biol 19: 397-422, 2003.

15. Claas C, Stipp CS and Hemler ME: Evaluation of prototype transmembrane 4 superfamily protein complexes and their relation to lipid rafts. J Biol Chem 276: 7974-7984, 2001.

16. Yarden Y and Sliwkowski MX: Untangling the ErbB signalling network. Nat Rev Mol Cell Biol 2: 127-137, 2001.

17. Iwata S, Kobayashi H, Miyake-Nishijima R, Sasaki T, SoutaKuribara A, Nori M, Hosono O, Kawasaki H, Tanaka H and Morimoto C: Distinctive signaling pathways through CD82 and beta1 integrins in human T cells. Eur J Immunol 32: 1328-1337, 2002 .
18. Sugiura T and Berditchevski F: Function of alpha3beta1tetraspanin protein complexes in tumor cell invasion. Evidence for the role of the complexes in production of matrix metalloproteinase 2 (MMP-2). J Cell Biol 146: 1375-1389, 1999.

19. Odintsova E, Sugiura T and Berditchevski F: Attenuation of EGF receptor signaling by a metastasis suppressor, the tetraspanin CD82/KAI-1. Curr Biol 10: 1009-1012, 2000.

20. van Echten G and Sandhoff K: Ganglioside metabolism. Enzymology, Topology, and regulation. J Biol Chem 268: 5341-5344, 1993.

21. Huwiler A, Kolter T, Pfeilschifter J and Sandhoff K: Physiology and pathophysiology of sphingolipid metabolism and signaling. Biochim Biophys Acta 1485: 63-99, 2000.

22. Hakomori S: Glycosphingolipids in cellular interaction, differentiation, and oncogenesis. Annu Rev Biochem 50: 733-764, 1981.

23. Hakomori S: Tumor malignancy defined by aberrant glycosylation and sphingo(glyco)lipid metabolism. Cancer Res 56: 5309-5318, 1996

24. Birklé S, Zeng G, Gao L, Yu RK and Aubry J: Role of tumorassociated gangliosides in cancer progression. Biochimie 85: 455-463, 2003

25. Regina Todeschini A and Hakomori SI: Functional role of glycosphingolipids and gangliosides in control of cell adhesion, motility, and growth, through glycosynaptic microdomains. Biochim Biophys Acta 1780: 421-433, 2008.

26. Hakomori SI: Glycosynaptic microdomains controlling tumor cell phenotype through alteration of cell growth, adhesion, and motility. FEBS Lett 584: 1901-1906, 2010.

27. von Lindern JJ, Rojo D, Grovit-Ferbas K, Yeramian C, Deng C, Herbein G, Ferguson MR, Pappas TC, Decker JM, Singh A, et al: Potential role for CD63 in CCR5-mediated human immunodeficiency virus type 1 infection of macrophages. J Virol 77: 3624-3633, 2003.

28. Park S-Y, Yoon S-J, Freire-de-Lima L, Kim J-H and Hakomori SI: Control of cell motility by interaction of gangliosides, tetraspanins, and epidermal growth factor receptor in A431 versus KB epidermoid tumor cells. Carbohydr Res 344: 1479-1486, 2009.

29. Todeschini AR, Dos Santos JN, Handa K and Hakomori SI: Ganglioside GM2/GM3 complex affixed on silica nanospheres strongly inhibits cell motility through CD82/cMet-mediated pathway. Proc Natl Acad Sci USA 105: 1925-1930, 2008.

30. Birchmeier C, Birchmeier W, Gherardi E and Vande Woude GF: Met, metastasis, motility and more. Nat Rev Mol Cell Biol 4: 915-925, 2003.

31. Tringali C, Silvestri I, Testa F, Baldassari P, Anastasia L, Mortarini R, Anichini A, López-Requena A, Tettamanti G and Venerando B: Molecular subtyping of metastatic melanoma based on cell ganglioside metabolism profiles. BMC Cancer 14: $560,2014$.

32. Tanaka K, Miyazawa M, Mikami M, Aoki D, Kiguchi K and Iwamori M: Enhanced expression of unique gangliosides with GM2-determinant in human uterine cervical carcinoma-derived cell lines. Glycoconj J 33: 745-754, 2016.

33. Ladisch S and Gillard B: A solvent partition method for microscale ganglioside purification. Anal Biochem 146: 220-231, 1985.

34. Huang X, Li Y, Zhang J, Xu Y, Tian Y and Ma K: Ganglioside GM3 inhibits hepatoma cell motility via down-regulating activity of EGFR and PI3K/AKT signaling pathway. J Cell Biochem 114: 1616-1624, 2013.

35. Leibovitz A, Stinson JC, McCombs WB III, McCoy CE, Mazur KC and Mabry ND: Classification of human colorectal adenocarcinoma cell lines. Cancer Res 36: 4562-4569, 1976.

36. Davies MA: The role of the PI3K-AKT pathway in melanoma. Cancer J 18: 142-147, 2012.

37. Mook OR, Frederiks WM and Van Noorden CJ: The role of gelatinases in colorectal cancer progression and metastasis. Biochim Biophys Acta 1705: 69-89, 2004.

38. Mueller KL, Powell K, Madden JM, Eblen ST and Boerner JL: EGFR tyrosine 845 phosphorylation-dependent proliferation and transformation of breast cancer cells require activation of p38 MAPK. Transl Oncol 5: 327-334, 2012.

39. Li Y, Huang X, Zhang J, Li Y and Ma K: Synergistic inhibition of cell migration by tetraspanin CD82 and gangliosides occurs via the EGFR or cMet-activated P13K/Akt signalling pathway. Int J Biochem Cell Biol 45: 2349-2358, 2013. 